Original Paper http://ajol.info/index.php/ijbcs $\quad$ http://indexmedicus.afro.who.int

\title{
Assessment of the validity of rapid diagnostic test kits available in the Nigerian market for Mycobacterium tuberculosis
}

\author{
R.C. CHUKWUANUKWU ${ }^{1 *}$, S.M. ODIDO ${ }^{1}$, C.C. ONYENEKWE ${ }^{1}$, E. ANYABOLU ${ }^{2}$, \\ M.C. ONWUNZO ${ }^{3}$, R.O. OKONKWO ${ }^{3}$, M.O. IFEANYICHUKWU ${ }^{1}$, C.O. OKEKE ${ }^{1}$ ' \\ and F. IFEDIATA ${ }^{4}$
}

\author{
${ }^{I}$ Medical Laboratory Science Department, Nnamdi Azikiwe University, Nnewi Campus, Nnewi. \\ ${ }^{2}$ Medicine Department, $\quad$ Nnamdi Azikiwe University Teaching Hospital, Nnewi \\ ${ }^{3}$ Microbiology Department, Nnamdi Azikiwe University Teaching Hospital, Nnewi \\ ${ }^{4}$ Haematology Department, Nnamdi Azikiwe University Teaching Hospital, Nnewi. \\ "Corresponding author; E-mail: beckytchuks@yahoo.com
}

\begin{abstract}
Tuberculosis (TB) is an infectious disease caused by various strains of mycobacteria, usually Mycobacterium tuberculosis. Early diagnosis is important in TB disease control. The use of rapid diagnostic test (RDT) kits drastically reduces the time required for reaching clinical diagnosis and this has been successful in diagnosis of HIV, syphilis and more recently malaria to mention a few. What is the case for RDTs for diagnosis of tuberculosis? Thus, the aim of this study is to evaluate and compare the performances of results produced by different rapid diagnostic test strips available in the Nigerian market for TB. A total of one hundred and eighty-four (184) subjects aged between 12-68 years were recruited for the study. These were referred from the chest clinic with a high index of suspicion of TB. Sputum samples were collected for AFB detection using the Ziehl-Neelsen and Auramine-phenol staining techniques. Blood samples were collected for serology tests using five (5) different rapid diagnostic test kits from different manufacturers, HIV status determination and evaluation of the haematological parameters we carried out. As a result, there were significant differences in the results obtained between AFB tests and serological methods with $\mathrm{P}<0.01$ in all cases. The sensitivity and specificity respectively of the five different kits were $28.6 \%$ and $32.0 \%$ (Nova), $19.5 \%$ and $29.2 \%$ (Fistech), $9.1 \%$ and $27.1 \%$ (Diaspot), $12.4 \%$ and $27.7 \%$ (Abcon) and $12.4 \%$ and $27.7 \%$ (Global).finally, findings from this study show that these rapid serological tests are poor in diagnosing tuberculosis and cannot be recommended for use in this environment.
\end{abstract}

() 2015 International Formulae Group. All rights reserved.

Keywords: Tuberculosis, rapid diagnostic tests, acid fast bacilli.

\section{INTRODUCTION}

Tuberculosis (TB) is a common and in many cases lethal, infectious disease caused by various strains of mycobacterium, usually Mycobacterium tuberculosis (Kumar et al., 2007). One third of the world's population is thought to have been infected with $M$. tuberculosis and in 2013, there were an estimated 9 million new cases and 1.5 million associated deaths with $95 \%$ of the deaths occurring in low and middle income countries (WHO, 2014). More people in the developing 
world are thought to contract tuberculosis because of compromised immunity (Lawn and Zumla, 2011), the high rates of HIV infection and the corresponding development of AIDS which is contributing to this burden and has led to a syndemic (Kwan and Ernst, 2011).

At present, TB diagnosis in most developing countries relies largely on clinical features, x-ray, and microscopy of acid-fast sputum stains. Cultivation of the organism is the diagnostic gold standard in which the organism is cultured for weeks and there is also the possibility of susceptibility testing for the organisms which grow. This improves treatment efficiency, however, the turn-around time before a definite diagnosis can be obtained is 3-6 weeks, and facilities for culture are not readily available (Aderemi and Dada-Adegbola, 2013), even in tertiary health facilities. In high-incidence countries, TB control relies on passive case finding among individuals self-presenting to health care facilities, followed by either diagnosis based on clinical symptoms and/or laboratory diagnosis using sputum smear microscopy (Parsons et al., 2011). Serial sputum specimens are required (one taken on the spot and the second brought in the following morning), which means that the people are asked to make repeated visits to the health care center for specimen delivery and collection of results. For many patients, the costs of repeated visits to health care facilities are prohibitive, and patient dropout is a significant problem. In addition, the sensitivity of sputum smear microscopy has been reported to vary often depending on the diligence with which specimens are collected, smears are made, and stained smears are examined (Steingart et al., 2007). Also, TB smear microscopy is highly insensitive for HIV co-infected individuals and for children due to the reduced pulmonary bacillary loads in these patients (Getahun et al., 2007). In resource-poor countries, many smear microscopy laboratories are single-room and understaffed with poorly maintained microscopes, and some of these laboratories lack consistent sources of electricity and clean water. There are few opportunities for the training of staff and little staff capacity to handle high-volume workloads. Quality assurance programs including quality control and external quality assessments (EQAs) are often lacking. Thus, there is a critical need for new, sensitive, easy, and rapid point-of-care diagnostics and also for investments in laboratory infrastructure, quality assurance programs, and well-trained staff (Parsons et al., 2011).

Timely diagnosis is crucial to $\mathrm{TB}$ control, as it provides early initiation of therapy and limits further disease spread. Several diagnostic methods for detecting TB have been used over the years including sputum smears and sputum culture. The tuberculin skin test (mantoux) for detection of latent TB is also in use. In addition, chest $\mathrm{x}$ rays are used to aid clinical diagnosis. All these methods have limitations. Newer tests, such as PCR-DNA amplification for TB diagnosis or interferon- gamma release assays for latent TB have been recently introduced. These tests are quite expensive and not practical for developing countries (Lyashchenko et al., 2000), due to their high cost and larger population with the disease burden. Rapid serological methods are an attractive alternative, since TB serodiagnosis is simple, inexpensive, relatively noninvasive, and it does not depend on detection of mycobacteria (Lyashchenko et al., 2000). By using Serodiagnostic methods, the time required for reaching a clinical decision to treat a suspected case of tuberculosis can be drastically reduced, as in some cases the actual proof of an organism's identity does not depend on its isolation or identification in a specific culture (Good and Mastro, 1989). Serodiagnosis is a rapid technique that is technically facile and if in routine use, is inexpensive. According to manufacturers of these rapid test kits for $\mathrm{TB}$, the technique is also usable for diagnosing non pulmonary TB and is particularly attractive for the identification of TB manifestations in which 
specimens are not easily accessible, example, skeletal TB. These rapid serological tests for TB are designed with antigens which are specific to Mycobacterium tuberculosis (Reddy et al., 2002).

The TB Rapid Test Device (whole blood/serum/plasma) is a rapid test for qualitative detection of anti - TB antibodies (Isotypes $\operatorname{IgG}, \operatorname{IgM}$ and $\operatorname{IgA}$ ) in whole blood, serum or plasma specimens. The test utilizes a combination of recombinant antigens to detect elevated levels of anti - TB antibodies in whole blood, serum or plasma specimens. These rapid diagnostic test kits for tuberculosis made by various manufacturers are readily available in Nigeria. There are conflicting reports of the sensitivity and specificity of these RDTs in research carried out in India with a very wide range of $1 \%$ $98.5 \%$ reported and sensitivity ranging from 53-99\% (Reddy et al., 2002; Steingart et al., 2012). Despite these reports, and the fact that no international guidelines recommend their use (Steingart et al., 2012), these RDTs are still very much in use especially in the private sector in many high burden countries including Nigeria (Greiner et al., 2012).

Quality assurance and control of rapid immunochromatographic rapid diagnostic test strips are not usually carried out in Nigeria to ascertain the sensitivity and specificity of these test strips/cassettes. Indeed proper regulation of laboratory reagents and consumables is lacking. This has led to free importation of both standard, sub standard and out rightly fake products by marketers. This in turn could lead to wrong results which are detrimental to patient treatment and well being. In addition to the inherent limitations of the test kits themselves, some of these rapid test strips may give less than satisfactory results due to environmental constraints, poor storage conditions leading to either false positive, false negative or invalid results. The net effect is wrong diagnosis and mismanagement of the patient.
This research was then designed to assess the validity, performance and reproducibility of some rapid immunochromatographic MTB-specific antibody detection tests using AFB positive patients who are not yet on anti-TB drugs. The study evaluated and compared the performances and results produced by five of the most popular rapid diagnostic test strips available in the Nigerian market by comparing results obtained with that in patients who were diagnosed by AFB and clinical assessment (in the absence of culture which is the gold standard). Due to the relationship between HIV and TB, HIV co-infected subjects were not excluded so long as they test positive for AFB. However, HIV positive individuals who tested negative for AFB were excluded because of the low sensitivity of AFB in HIV.

\section{MATERIALS AND METHODS}

\section{Subjects}

A total number of 184 subjects (males and females) aged between 12 years to 68 years were recruited from the tuberculosis directly observed treatment short course (TB DOTS) clinic, Nnamdi Azikiwe University Teaching hospital, Nnewi. These were suspected cases of tuberculosis referred from the chest clinic after clinical assessment of presenting symptoms and examination of chest X-rays.

\section{Exclusion/inclusion criteria}

TB positive patients who were already on anti-TB drugs were excluded from the study. Only freshly diagnosed treatment naïve TB patients were selected for the study.

\section{Ethical consideration}

The research design was approved by the Ethical committee of Nnamdi Azikiwe University Teaching Hospital Nnewi. Informed consent was obtained from each of the participants. Confidentiality was maintained. 
Information such as age and history of infection was obtained from the subjects by questionnaires.

\section{Sample collection}

Blood samples used were Ethylene diamine tetra-acetic acid (EDTA) anticoagulated blood (for estimation of the packed cell volume (PCV) and white blood cell count (WBC) and clotted blood (for serological detection of TB antibodies and HIV Screening) collected by venepuncture technique from the antecubital vein. Three sputum samples (for the Ziehl-Neelsen and Auramine-phenol techniques) were collected using a clean, sterile, wide-mouthed universal container. Both Ziehl-Neelsen and Auramine phenol techniques were used for each sample for AFB testing.

\section{Ziehl-Neelsen technique for M. tuberculosis}

The Ziehl-Neelsen technique is used to stain Mycobacterium species. Mycobacteria, unlike most others, do not stain well by the Gram technique. They can however be stained with carbol fuchsin combined with phenol. The stain binds to the mycolic acid in the mycobacterial cell wall. After staining, an acid decolourizing solution is applied. This removes the red dye from the background cells, tissue fibres, and any organisms in the smear except mycobacteria retain (hold fast to) the dye and therefore referred to as acid fast bacilli or simply AFB. Following decolourization, the smear is counterstained with methylene blue which stains the background material, providing a contrast colour against which the red AFB can be seen (Cheesbrough, 2002). Sputum smears were made on clean grease free slides and procedure was according to standard laboratory operating procedure.

\section{Auramine-Phenol technique}

Auramine fluoresces when illuminated (excited) by violet or ultra-violet (UV) light. It can be used to demonstrate AFB because it binds to the mycolic acid in the mycobacterial cell wall. No heating of the stain is required. After being stained with auramine, the smear is decolorized with acid alcohol which removes the dye from the background. The smear is then washed with a weak solution of potassium permanganate to darken the background. TB fluoresces white-yellow against a dark background (Cheesbrough, 2002). Sputum smears were made on clean grease free slides and procedure was according to standard laboratory operating procedure.

For both AFB methods, the numbers of bacteria present were reported per high power field as follows:

More than 10 AFB/field: +++

1-10 AFB/field: ++

10-1000 AFB/100 fields: +

1-9 AFB/100 fields: exact number was reported.

Although most AFB sputum smear positive patients are indeed infected with $M$. tuberculosis (Idigbe et al., 1986), samples can be contaminated with environmental mycobacteria and this is often seen in stored samples (Salvakumar et al., 2006). To overcome this, all samples were examined by experienced microscopists with 24 hours of smear preparation. Freshly prepared stains were used and standard staining procedures were followed.

\section{HIV screening}

Two different methods were used namely: Inverness Determine ${ }^{\mathrm{TM}} 1$ \& 2 (Inverness Medical Japan Co. Ltd, Japan) and STAT-PAK (Chembio Diagnostic system, New York, USA). HIV seropositive results using these two methods are used to classify participants as HIV infected. Tests were carried out according to manufacturer's instructions.

Appearance of two distinct colored lines, one at the control region and the other at the test region indicates a positive result while 
appearance of one colored line at the control region indicates a negative result.

\section{TB antibodies serological testing}

Five different rapid test kits were purchased from suppliers who import and distribute these products in the country. They are all based on the same principle of detection of antibodies to tuberculosis. The TB Tuberculosis Rapid Test Device (Whole blood/serum/plasma) is a qualitative, solid phase, two site sandwich immunoassay for the detection of anti-TB antibodies in whole blood, serum or plasma specimens. The membrane is pre-coated with TB recombinant antigen on the test line region of the device. During testing the anti-TB antibodies, if present in whole blood, serum or plasma specimen react with the particles coated with TB recombinant antigen. The mixture migrates upward on the membrane chromatographically by capillary action to react with $\mathrm{TB}$ recombinant antigen on the membrane and generate a colored line. The presence of this colored line in the test region indicates a positive result, while its absence indicates a negative result. To serve as a procedural control, a colored line will always appear in the control line region indicating that proper volume of specimen has been added and membrane wicking has occurred (Bothamley, 1995). Appearance of two distinct colored lines, one at the control region and the other at the test region indicates a positive result while appearance of one colored line at the control region indicates a negative result. The intensity of the color at the test region varies depending on the concentration of anti-TB antibodies present in the specimen. All test procedures were carried out according to the different manufacturers' instructions. Serum samples were used for all analyses.

The packed cell volume and total white blood cell counts were done by manual methods as described by Bain et al., 2008, using standard operating procedures.

\section{Statistical analysis}

Data were analyzed using Graph pad Prism version 5.03. Spearman's correlations, Pearson's Chi-Square were used. Sensitivity and specificity for the diagnostic method were performed. Positive (PPV) and negative (NPV) predictive values as well as likelihood ratios were calculated. The level of significance was set at $P<0.05$.

\section{RESULTS}

A total of 184 subjects referred from the chest clinic participated in the study. Of these 120 were TB positive subjects who were positive for $\mathrm{TB}$ via the sputum smear microscopy. The mean PCV (L/L) were 38.11 +3.37 and $40.22+6.78$ in the AFB positive subjects and negative subjects respectively. The mean WBC counts $\left(10^{9} / 1\right)$ were $6.17+$ 2.38 and $5.67+0.85$ in the AFB positive subjects and negative subjects respectively (Table 1). Serum samples from each AFB positive and negative subject was subjected to further testing using all five RDTs and the results are shown in table 2 . The mean sensitivity and $95 \%$ confidence interval (C.I) are $12.4 \%$ (C.I=7.3-19.1) for Abcon, 9.1\% (C.I=4.7-15.3) for Diaspot, $19.5 \%$ (C.I= 13.426.7) for Fistech, 12.4\% (C.I=7.3-19.1) for Global and $28.6 \%$ (C.I=21.8-36.0) for Nova. The specificity and $95 \%$ confidence interval are $27.7 \%$ (C.I=22.0-33.9) for Abcon, 27.1\% (C.I=21.6-33.3) for Diaspot, 29.2\% (C.I= 23.3-35.7) for Fistech, $27.7 \%$ (C.I=22.0-33.9) for Global and 32\% (C.I=25.6-38.9) for Nova. The negative predictive values, positive predictive values and likelihood ratios are shown in table 2 .

There were no significant differences in the PCV $(\mathrm{P}=0.451)$ and WBC $(\mathrm{P}=0.561)$ values among the subjects who are false negative with the RDTs and those who are true positives. Thus, these do not have any effect on the test results produced by these kits.

Of the $120 \mathrm{~TB}$ positive test subjects, forty-five (45) of them were HIV positive 
$(37.5 \%)$ while seventy-five (75) of the subjects $(62.5 \%)$ were HIV negative (Figure 1). None of the TB negative subjects included in the study was positive for HIV.

Among the HIV positive individuals who were AFB positive, $31.3 \%$ tested positive in one or more test kits while among the AFB positive subjects who were not co-infected with HIV, $46.1 \%$ tested positive in one or more test kits. The sensitivity relationship of the RDTs with HIV positive status (immunocompromised) calculated is $6.3 \%$ for Nova, $13 \%$ for Fistech, $6.3 \%$ for Diaspot, $13 \%$ for Abcon and $19 \%$ for Global while the sensitivity relationship with HIV negative status gave $44 \%$ for Nova, $24 \%$ for
Fistech, $12 \%$ Diaspot,16\% for Abcon and 8\% for Global. Thus, HIV infection affects the performance of the test kits. In addition, there was a weak positive correlation in the performance of the test kits when comparing mycobacterial load (as shown by the number of mycobacteria observed microscopically) and HIV infection $(r=0.373)$, thus, with higher mycobacterial load, detection in the HIV coinfected increases weakly. On the other hand, in the HIV negative, there was no clear relationship (in the 5 different makes of the RDTs) between the mycobacterial density and the performance of the test kits.

Age and sex did not appear to have any effect on performance of the test kits.

Table 1: Sputum smears, age, sex, packed cell volume (PCV) and Total white blood cell (TWBCT) counts in the subjects.

\begin{tabular}{lclcc}
\hline $\begin{array}{l}\text { SPUTUM SMEARS } \\
\text { (AFB TEST) }\end{array}$ & $\begin{array}{c}\text { Mean age } \\
(+ \text { S.D) }\end{array}$ & \multicolumn{1}{c}{ Sex } & $\begin{array}{c}\text { PCV(L/L) } \\
(+ \text { S.D) }\end{array}$ & $\begin{array}{c}\text { TWBC(10 }) \\
(+ \text { S.D) }\end{array}$ \\
\hline $\begin{array}{l}\text { AFB positive } \\
(\mathrm{n}=120)\end{array}$ & $36.5+13.5$ & $\begin{array}{l}\text { Male: 69(57.5\%) } \\
\text { Female: } 51(42.5 \%)\end{array}$ & $38.1+3.4$ & $6.2+.38$ \\
\hline $\begin{array}{l}\text { AFB negative } \\
(\mathrm{n}=64)\end{array}$ & $39.2+15.6$ & $\begin{array}{l}\text { Male: 31(48.4) } \\
\text { Female: } 33(51.6)\end{array}$ & $40.2+0.78$ & $5.7+0.85$ \\
\hline Total (184) & & & & \\
\hline
\end{tabular}

Table 2: Sensitivity, specificity, positive predictive value (PPV), negative predictive value (NPV) and likelihood ratios of the serology tests of rapid TB test kits. Confidence intervals (C.I) are also shown.

\begin{tabular}{lccccc}
\hline$\%$ & ABCON & DIASTOP & FISTECH & GLOBA & NOVA \\
\hline Sensitivity & 12.2 & 9.1 & 19.5 & 12.4 & 28.6 \\
95\% C.I & $7.4-19.1$ & $4.7-15.3$ & $13.4-26.7$ & $7.4-19.1$ & $21.8-36.0$ \\
\hline Specificity & 27.7 & 27.1 & 29.2 & 27.7 & 32.0 \\
95\% C.I & $22.0-33.9$ & $21.6-33.3$ & $23.3-35.7$ & $22.0-33.9$ & $25.6-38.9$ \\
\hline PPV & 9.2 & 6.5 & 15 & 9.2 & 26 \\
95\% C.I & $5.5-14.4$ & $3.4-11.1$ & $10.8-21.8$ & $5.4-14.4$ & $19.9-33.0$ \\
\hline NPV & 34.8 & 34.8 & 34 & 34.8 & 34.8 \\
95\% C.I & $27.9-42.1$ & $27.7-42.1$ & $27.9-42.1$ & $27.9-42.1$ & $27.9-42.1$ \\
\hline \multicolumn{7}{c}{ (Not in \%) Likelihood } \\
\hline ratio & 0.17 & 0.12 & 0.28 & 0.17 \\
\hline
\end{tabular}




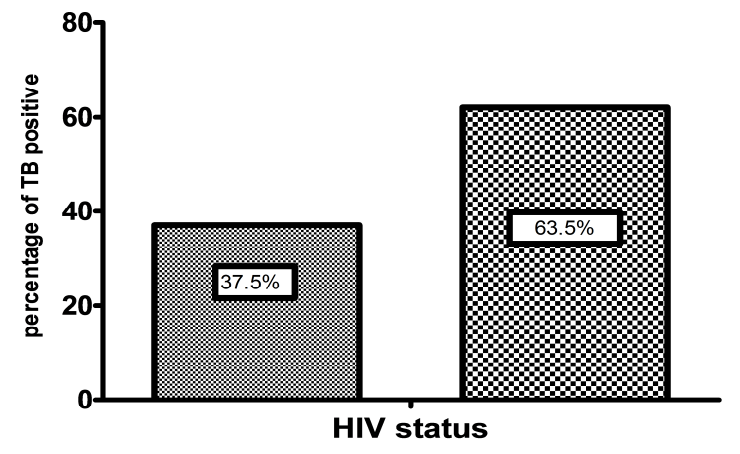

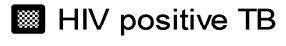

8 HIV negative TB

Figure 1: The HIV status of TB positive subjects.

\section{DISCUSSION}

Though newer methods of TB detection such as nucleic acid amplification-based diagnostic systems are highly accurate and precise and can provide a definite diagnosis within hours, high cost is a barrier for resource poor countries to scale up (Boehme et al., 2010). Most patients have access to only smear microscopy and even this is under utilized in the private sector (Jaroslawski and Pai, 2012). Rapid serological tests have been used successfully in the diagnosis of many infectious diseases including HIV and viral hepatitis and have been available for decades. The ease of performing these tests as well as time it takes to reach a diagnosis certainly makes these rapid diagnostic tests very attractive. Rapid serologic tests for detection of active TB have been commercially available for decades (Steingart, 2012) and though no international guidelines recommend their use, dozens of these commercial test kits are offered for sale in many parts of the world (Jaroslawski and Pai, 2012) and there is a thriving market for them. These serological tests are widely used in high-burden countries including Nigeria (Greiner et al., 2012). An estimated 1.5 million serological TB tests are performed in India alone every year mostly in the private sector (Greiner et al., 2012). The exact estimate for Nigeria is unknown. With reports that these serological tests are inaccurate yet widely used (Steingart, 2012; Greiner et al., 2012; Jaroslawski and Pai, 2012) the validity of the test kits available for use in Nigeria were assessed.

Findings from this study show that the sensitivity and specificity of these kits are very low with Nova that gave the best result giving a sensitivity of $28.6 \%$ and specificity of $32 \%$. The result of our study does not agree with the findings of Reddy et al., 2012 carried out in India. They reported specificity of $99.42 \%$ with sensitivity of $98.52 \% \quad(n=241)$ and in cases of multiple infections, the specificity was $93.15 \%$ with a lower sensitivity of $73.52 \%(n=141)$. This does not conform to the findings of this study though the authors used a particular kit developed by them. However, the findings from this study are in accordance with reports by Steingart et al., 2012 from India which reviewed different TB serology kits. Their study on the performance of rapid diagnostic tests for pulmonary tuberculosis gave sensitivity results ranging from $1 \%$ to $59.71 \%$ and specificity ranging from $57.05 \%$ to $98.66 \%$. The two studies also identified that evaluated HIV-infected TB patients test sensitivities were poor most likely due to their immune state and the poor production of antibodies which are to be detected via this method. The sensitivity and specificity with all five serology test kits used in this study are low 
and one may argue that these were not purchased directly from the manufacturers, and as such poor storage may be an issue. That maybe true but only to an extent because that is the reality we face. Hardly any end user purchases these and other such test kits including the highly successful ones directly from the manufacturers, so these test results are a representation of diagnostic test results especially from the private sector where these rapid tests are mostly in use.

It was expected that the higher the mycobacterial density (number of $+s$ ) produced from the AFB smear microscopy method in HIV negative subjects the higher the production of true positives from the kits. This was not the case either in the HIVnegative or HIV positive group. Neither age nor sex appears to affect the performance of these kits.

The basic premise of serological tests is ease, rapidity and ever increasing demand in endemic countries. Hence, these rapid test kits have always been the first choice for small time laboratories that may be operating in rural areas without electricity and qualified man power. The use of these RDTs in the accurate diagnosis of HIV has been a major boost for these kits in recent years, however, unlike the RDTs for HIV and malaria detection, there has been no clear encouragement to use these RDTs for diagnosis of TB, yet the market for these kits are thriving. It has been reported that in some places, these kits are utilized without bothering much on quality of tests and implications of false-positive and falsenegative results (Singh and Katoch., 2011). These kits are not reliable for the diagnosis of $\mathrm{TB}$ in this environment with the highest sensitivity value of $28.6 \%$ from the Nova test kit brand which is very low in terms of sensitivity and specificity for diagnosis. The data from this study, as well as data from other studies and WHO evaluation (WHO,
2006) unambiguously show that TB serology should not be used for TB diagnosis. The idea that serology is cheaper has no basis, as thorough serology profile (IgG, $\operatorname{IgA}, \operatorname{IgM}$ ) costs more than even liquid culture and PCR test combined (Singh and Katoch.,2011). There is no evidence that existing commercial serological assays improve patient-important outcomes, and high proportions of falsepositive and false-negative results adversely impact patient safety (Steingart et al., 2012). Misdiagnosis of TB is harmful both to individual patients and to public health; every missed TB diagnosis may result in additional TB transmission (Greiner et al., 2012). It is therefore, recommended that these tests should not be used in individuals suspected of active pulmonary or extrapulmonary TB, irrespective of their HIV status (Steingart et al., 2011). Findings from this study lead us to agree with these statements. The search for appropriate serological diagnostic test kits for tuberculosis will continue, and till such a time that it is inferred from appropriate quality control tests that such is available, use of these available test kits can hardly be recommended. With these finding, it is recommended that local policy advising against the use of these serological test for TB should be made.

\section{Conclusion}

Currently available commercial serological tests for TB are not reliable. The present situation where laboratory consumables are not regulated and quality control to assess accuracy and precision are not in place can only do more harm than good. Appropriate legislation should be put in place urgently to address these lapses. More funding is advocated in order to provide the relatively new molecular diagnostic equipment such as the PCR GeneXpert and Interferon release assays which are accurate and fast in order to tackle the high tuberculosis disease burden. 


\section{ACKNOWLEDGEMENTS}

The authors wish to acknowledge the assistance of the clinical staff of TB DOTS clinic, Nnamdi Azikiwe University Teaching Hospital, Nnewi.

\section{REFERENCES}

Aderemi OK, Dada-Adegbola H. 2013. Epidemiology of smear-Negative Tuberculosis in Ibadan, Nigeria. Afr. $J$ Infect Dis., 7(1): 14-17.

Bothamley GH.1995. Serological diagnosis of tuberculosis. European Respiratory Journal, 8: 676s-688s.

Cheesbrough M. 2002. District Laboratory Practice in Tropical Countries (Part 2). Low-price Edition. Cambridge University Press: Cambridge, United Kingdom.

Bain BJ, Bates I, Laffan MA, Lewis SM. 2008. Dacie and Lewis Practical Haematology. $\left(11^{\text {th }}\right.$ edn). Churchill Livingstone Elsevier: London.

Boehme CC, Nabeta P, Hillemann D et al. 2010. Rapid molecular detection of tuberculosis and rifampicin resistance. New Engl. Journal Med., 363: 10051015.

Getahun H, Harrington M, O'Brien R, Nunn P. 2007. Diagnosis of smear-negative pulmonary tuberculosis in people with HIV infection or AIDS in resourceconstrained settings: informing urgent policy changes. Lancet, 369: 20422049.

Good RC, Mastro TD. 1989. The modern mycobacteriology laboratory. How it can help the Clinician. Clin. Chest. Med., 10: 315-322.

Grenier J, Pinto L, Nair D et al. 2012. Widespread use of serological tests for tuberculosis: data from 22 high burden countries. Eur. Resp. Journal, 39(2): 502-505.
Idigbe EO, Anyiwo CE, Onwujekwe DI. 1986. Human pulmonary infections with bovine and typical mycobacteria in Lagos, Nigeria. J. Trop Med. Hygiene, 89(3): 143-148.

Jaroslawski S, Pai M. 2012. Why are inaccurate tuberculosis serological tests widely used in the Indian private sector? A root cause analysis. $J$ of Epidemiol and global health, 2(1): 3950.

Kwan CK, Ernst JD. 2011. HIV and Tuberculosis: a Deadly Human Syndemic. Clin. Microbiol. Rev., 24(2): 351-376.

Kumar V, Abbas AK, Fausto N, Mitchell RN. 2007. Robbins Basic Pathology $\left(8^{\text {th }}\right.$ edn). Saunders Elsevier: Philadelphia.

Lawn SD, Zumla AI. 2011. Tuberculosis. Lancet, 378 (9785): 57-72.

Lyashchenko KP, Singh M, Colangeli R, Gennaro ML. 2000. A multi-antigen print immunoassay for the serological diagnosis of infectious diseases. $J$. Immunol methods, 242: 91-100.

Parsons LM, Somoskövi A, Gutierrez C, Lee E, Paramasivan CN, Abimiku A, Spector S, Roscigno G, Nkengasong J. 2011. Laboratory Diagnosis of Tuberculosis in Resource-Poor Countries: Challenges and Opportunities. Clin. Microbiol. Rev., 24 (2): 314-350.

Reddy JR, Kwang J, Lechtenberg KF, Khan NC, Prasad RB, Chengappa MM. 2002. An immunochromatographic serological assay for the diagnosis of Mycobacterium tuberculosis. Comp Immunol. Microbiol., 25(1): 21-27.

Selvakumar N, Kumar V, Sivagamesundar S, Narayanan PR. 2006. Contamination of stored sputum AFB smears with environmental mycobacteria. International Journal of Tuberculosis and Lung Disease, 10(11): 1299-1301. 
Singh S, Katoch VM. 2011. Commercial serological tests for the diagnosis of active tuberculosis in India: Time for introspection. Indian J. Med. Res., 134(5): 583-587.

Steingart KR, Flores LL, Dendukuri N, Schiller I, Laal S, Ramsay A,Hopewell PC, Pai M. 2011. Commercial serological tests for the diagnosis of active pulmonary and extrapulmonary tuberculosis: an updated systematic review and metaanalysis. PLoS Medicine, 8(8): e1001062.

Steingart KR, Ramsay A, Pai M. 2007. Optimizing sputum smear microscopy for the diagnosis of pulmonary tuberculosis. Expert Rev Anti infect Ther., 5: 327-331.

Steingart KR, Ramsay A, David W, Dowdy C, Madhukar P. 2012. Serologicaltests for the diagnosis of active tuberculosis: relevance for India. Indian J. Med. Res., 135: 695-702.

World Health Organization. 2006. Diagnosis for tuberculosis: Global demand and market potential. WHO: Geneva. Available at http://www.who.int/tdr/ publications/en.

World health Organization 2014. Global tuberculosis Report 2014. Geneva, Switzerland. Retrieved on 07/05/2015. 